Revue des patrimoines

43 | 2021

Des écoles d'art académiques aux écoles d'art : des collections et des lieux, un patrimoine à valoriser

\title{
Le modèle vivant, patrimoine absent des écoles d'art académiques
}

The live model: a missing heritage of the art schools

\section{Morwena Joly-Parvex}

\section{(2) OpenEdition}

\section{Journals}

Édition électronique

URL : http://journals.openedition.org/insitu/30031

DOI : 10.4000/insitu.30031

ISSN : 1630-7305

Éditeur

Ministère de la Culture

Référence électronique

Morwena Joly-Parvex, "Le modèle vivant, patrimoine absent des écoles d'art académiques », In Situ

[En ligne], 43 | 2021, document 19, mis en ligne le 12 janvier 2021, consulté le 19 janvier 2021. URL :

http://journals.openedition.org/insitu/30031; DOI : https://doi.org/10.4000/insitu.30031

Ce document a été généré automatiquement le 19 janvier 2021.

\section{cc) () $९$}

In Situ Revues des patrimoines est mis à disposition selon les termes de la licence Creative Commons Attribution - Pas d'Utilisation Commerciale - Pas de Modification 4.0 International. 


\title{
Le modèle vivant, patrimoine absent des écoles d'art académiques
}

\author{
The live model: a missing heritage of the art schools
}

Morwena Joly-Parvex

1 L'intérêt porté au patrimoine des écoles d'art témoigne de notre fascination pour l'acte créateur, quelle que soit son époque. Si déambuler au milieu des moulages antiques ou feuilleter des portefeuilles de dessins matérialise la préparation à la création, il n'est pas certain que le public puisse bien saisir l'essence de la formation pédagogique d'une école d'art à travers son patrimoine conservé, de manière plus ou moins aléatoire selon leur histoire.

Estampes, dessins, moulages, rondes bosses, peintures nous renseignent bien sur l'environnement visuel de l'artiste en formation, voire sur ses premiers essais de création dans le cadre des exercices proposés. Cependant, l'exercice pédagogique primordial en France, au fondement des « écoles académiques» de province, demeure l'observation de la nature et plus particulièrement du corps humain. L'étude du modèle vivant est si essentielle au projet académique français que les notions de modèle nu et d'académie fusionnent dans le vocabulaire artistique. Ces écoles reprennent en cela la grande innovation au cœur de la création de l'Académie royale de peinture et de sculpture de Paris (1648) qu'est l'institution du modèle vivant, dont les Carrache, à Bologne, avaient notamment contribué à diffuser la pratique dans les ateliers à partir de leur Académie (1582). L'étude d'après le modèle vivant fonde la spécificité des écoles de dessin d'Ancien Régime qui seront autorisées à s'appeler " académiques ", et reste réservée à une élite d'artistes et d'amateurs au regard de la cinquantaine d'écoles de dessin qui sont fondées au cours du XVIII siècle $^{1}$.

Cette spécificité n'est pourtant matérialisée que par les dessins appelés "académies ", qui ont été souvent aléatoirement conservés. Dans les écoles d'art héritières des écoles académiques, ce sont davantage le "matériel » pédagogique en lui-même, comme les moulages ou les estampes, qui ont été conservés, et non les productions d'élèves. Ce fait est plus important qu'il n'y paraît à l'heure où la question de la conservation et de l'exposition du patrimoine des écoles d'art peut poser question en maints endroits. N'y 
a-t-il pas un risque de surévaluation de l'importance des modèles dans la formation des artistes français, alors que le fondement de l'apprentissage demeure l'observation du corps vivant?

4 Cette question est déjà au cœur des préoccupations des professeurs et amateurs qui animent l'Académie royale de peinture et de sculpture jusqu'à sa disparition en 1793. Les conférences qui s'y déroulent reviennent inlassablement sur la place à accorder à l'observation de la nature par rapport à l'imitation des œuvres anciennes. Au sein de l'École des beaux-arts, tout au long du XIx ${ }^{e}$ siècle, le débat se poursuit. À la faveur du développement des moulages, les modèles anciens s'accumulent au sein des écoles d'art, même si parallèlement on cherche à retrouver l'étude de la nature à travers le dessin du corps disséqué. La matérialité du corps vivant, avec ses "défauts » et sa puissante réalité, a toujours à la fois posé problème et fasciné les recherches artistiques.

5 La confrontation de la nature et des modèles constitue bien le cœur de la réflexion pédagogique au sein des écoles d'art, et ne montrer que les modèles, majoritairement gréco-romains, parfois classiques et moins souvent datant de la Renaissance, biaise la réflexion pédagogique qui a été menée. Le patrimoine des écoles d'art ne prend sens que s'il est compris dans son rapport au corps vivant et sa matérialité, bien souvent éloignée des canons de la plastique gréco-romaine. L'idée est ici de retracer l'histoire de ce « patrimoine » absent pourtant au cœur de la pédagogie artistique.

\section{Le cours d'après le modèle vivant au cœur des réflexions pédagogiques à l'époque classique}

$6 \quad$ L'Académie royale de peinture et de sculpture de Paris a fini par concentrer, au fil des années, un ensemble de privilèges qui rendent son rôle de plus en plus prépondérant, parmi lesquels le privilège exclusif de pouvoir " poser le modèle » jusqu'en $1706^{2}$, afin de se démarquer de la formation proposée par des corporations. Dès sa fondation en 1648, Charles Le Brun et d'autres artistes conçoivent une pédagogie fondée sur l'enseignement du dessin d'après modèle vivant ${ }^{3}$, à l'image de la première académie d'art qui ouvre à Florence en 1563.

7 L'Académie des Incamminati ouverte à Bologne en 1582 par les peintres Annibal, Agostino et Ludovico Carrache ${ }^{4}$, qui avait particulièrement développé cet exercice, constitue la deuxième référence majeure de Paris. Si la France emprunte tant aux Carrache, c'est que le préjugé contre le nu est devenu très puissant, et « on travaillait depuis tant de temps sur des copies de dessins de maîtres, qu'on avait perdu la notion de la nature ${ }^{5} »$. Or, aux yeux de Charles Le Brun, le travail d'après nature est essentiel car il prévient de la manière. En 1648, il offre à cet effet à l'Académie "plusieurs membres détachés, moulés en plâtre sur le cadavre d'un homme écorché ${ }^{~}$. L'accès à salle des modèles est strictement réglementé, et un " concours des places » est instauré afin d'organiser l'entrée à ce cours très prisé [fig. 1, fig. 2]. 


\section{Figure 1}

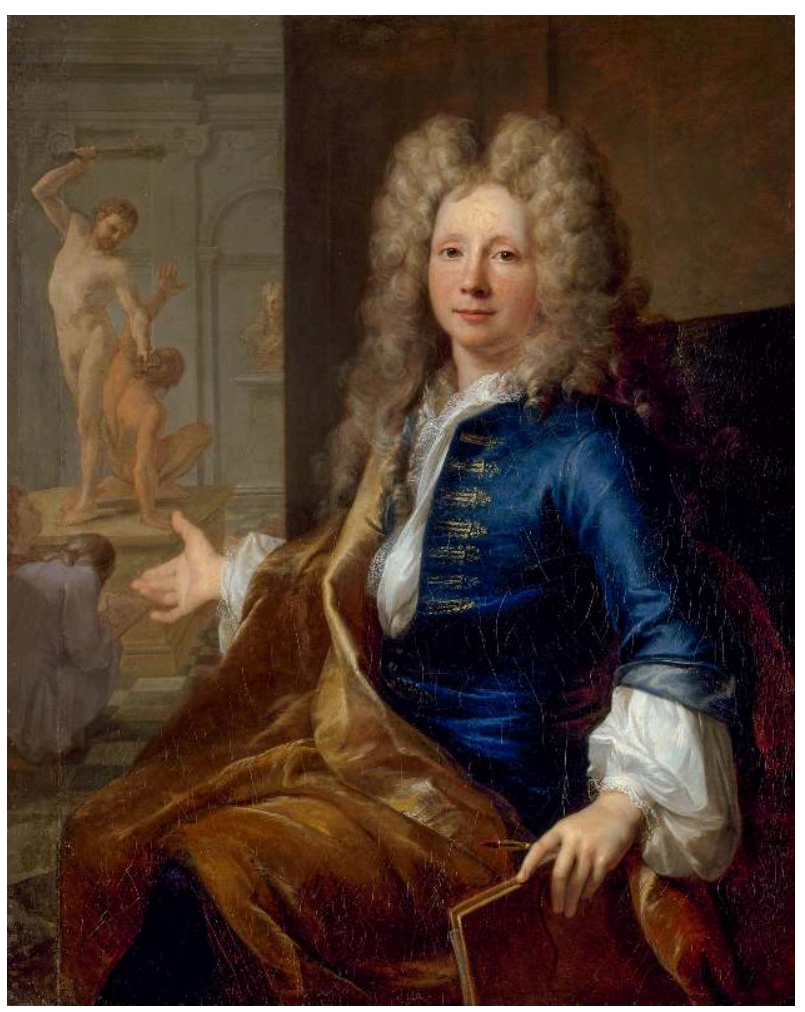

Portrait de Louis de Boullogne le jeune, peintre (1654-1733), devant une séance de pose d'après le modèle vivant à l'Académie royale de peinture, Pierre Gobert, huile sur toile $(115 \times 88 \mathrm{~cm})$ conservée au musée national des châteaux de Versailles et de Trianon (inv. MV5821 Versailles).

Reproduction Gérard Blot (RMN-Grand Palais). 
Figure 2

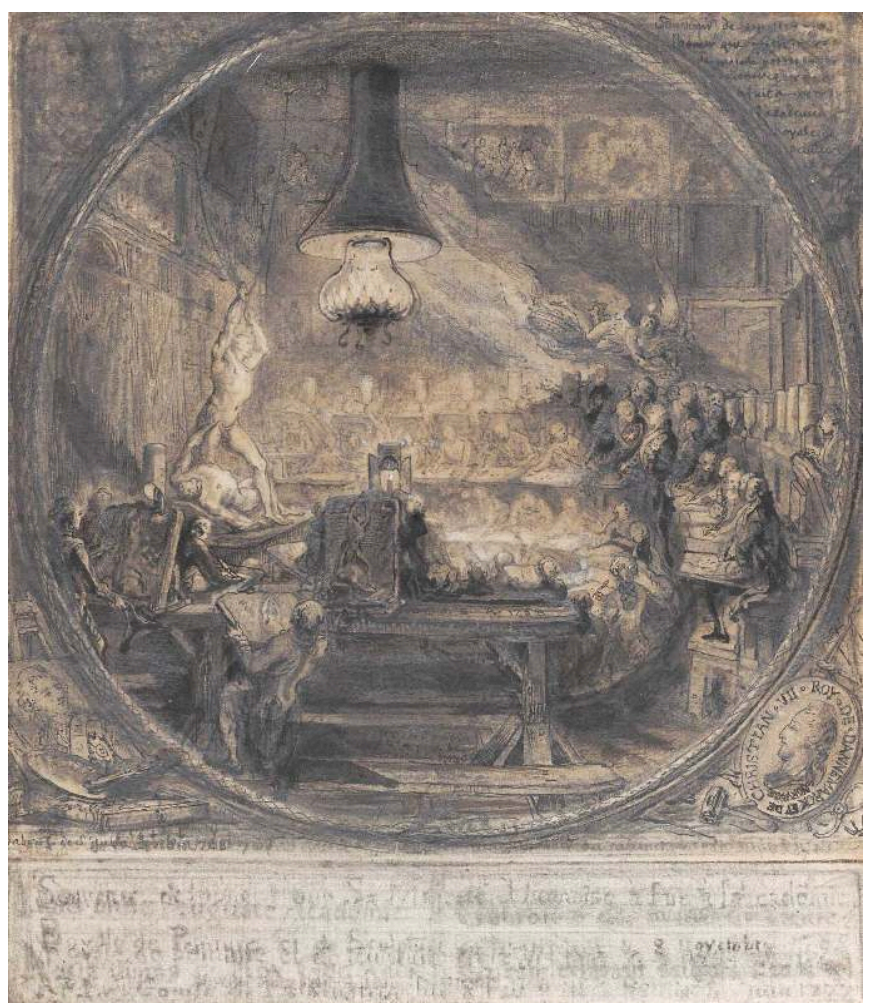

Visite du roi Christian VII à l'Académie de Paris, le 8 novembre 1768, Gabriel de Saint-Aubin (1724-1780), dessin (18,1 x 16 cm) conservé au Statens Museum for Kunst (inv. KKS1964-306), Copenhague.

Reproduction Statens Museum for Kunst.

Mais dès l'année 1667, alors qu'une série de conférences théoriques vient compléter l'enseignement pratique dispensé aux élèves, Roger de Piles, amateur influent au sein de l'institution, publie un Abrégé d'anatomie qui s'interroge sur la " routine du contour » que le dessin d'après le modèle vivant peut engendrer. C'est que l'observation stricte de la nature, au cœur de l'initiation artistique, pose la question du degré d'idéalisation dont personne ne conteste la nécessité à l'époque classique. L'esthétique classique prône en effet l'imitation d'une "belle nature ", et non d'une nature commune, au risque de tomber dans l'inexactitude anatomique.

Le dessin d'après le modèle vivant suscite de vifs débats au sein des académiciens : quelle place accorder au modèle antique, à Raphaël tant et tant copié, à la stricte observation du réel? À la fin $d u \mathrm{XVII}^{\mathrm{e}}$ siècle, on s'inquiète de la tentation de la "manière ", expression par trop singulière du réel héritée de Michel-Ange, puis, au milieu du XVIII ${ }^{e}$ siècle, une polémique plus structurée au sein des académiciens oppose les tenants du «Beau idéal » et ceux du « Beau réel ${ }^{7}$ » qui s'interrogent sur la place que doit tenir le modèle antique dans la formation artistique. Charles-Nicolas Cochin, alors secrétaire influent de l'Académie, craint que l'institution ne se transforme en fabrique de modèles dérivés de l'antique. En dépit de ces intenses débats, la question reste entière devant le corps vivant du modèle: comment conjuguer idéalisation et observation de la nature?

Faute de théorie esthétique consensuelle, la dénonciation de la «manière » dans le dessin continue d'être dénoncée par les amateurs, comme chez Diderot, en 1765 : « Il n'y aurait point de manière ni dans le dessin ni dans la couleur, si l'on imitait 
scrupuleusement la nature. La manière vient du maître, de l'Académie, de l'école et même de l'antique ${ }^{8}$. " Imiter "scrupuleusement la nature " ? Selon Diderot, le modèle vivant, avec ses imperfections et sa chair toujours en métamorphose, variant selon les émotions du sujet, constitue un défi majeur pour l'artiste.

Or l'usage veut que l'étude du modèle vivant ne vienne qu'après des exercices de copie, d'après des estampes ou des plâtres. Cette pratique s'est imposée dans toutes les écoles de dessin au motif que «le moindre mouvement involontaire et presqu'insensible dans le modèle vivant embarrasse le jeune artiste en lui présentant souvent des surfaces nouvelles ${ }^{9}$ ». Être capable de se confronter à la nature est considéré comme une épreuve qualifiante mais difficile sans l'exercice préalable de la copie. En Angleterre, William Hogarth livre en 1753 une version pleine d'humour des débats théoriques sur ce que serait une " analyse de la beauté » sans aucune référence au modèle vivant : une accumulation de formes antiques, de planches anatomiques, de têtes d'expression ou de formes courbes associées dans une joyeuse disparité [fig. 3].

Figure 3

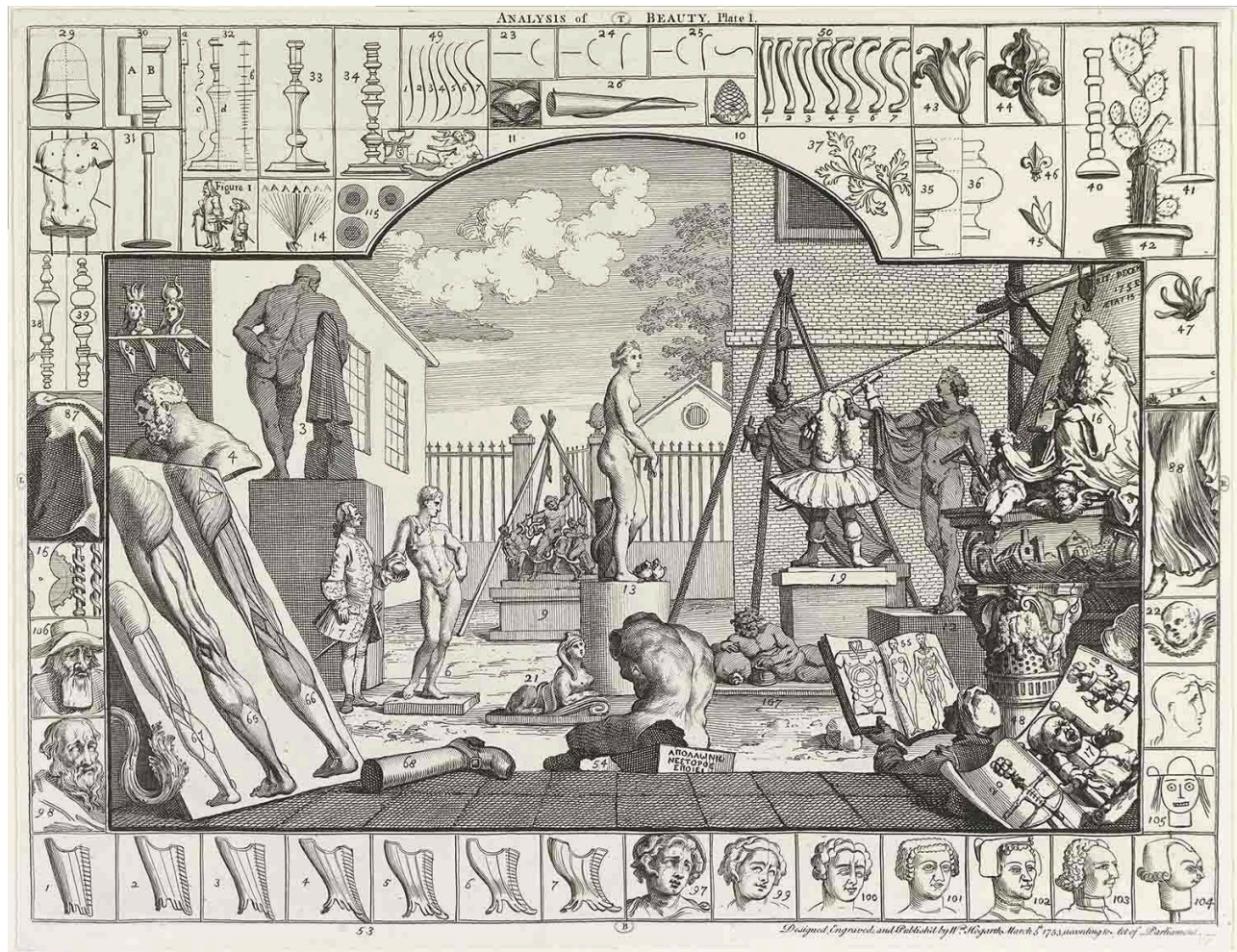

The Analysis of Beauty, William Hogarth (1697-1764), planche 1, 1753. Gravure à l'eau forte (38,8 x $50 \mathrm{~cm}$ ) conservée au Metropolitan Museum of Art (inv. 32.35 22), New York.

Reproduction Metropolitan Museum of Art.

Les estampes, des manuels de dessin ainsi que les rondes-bosses fréquemment conservés ne sont que des témoignages de la préparation à l'exercice du dessin d'après le modèle vivant. Encore faut-il souligner au sujet des estampes qu'aucune " méthode " n'est agréée de manière consensuelle à l'Académie de Paris, en dépit de la profusion d'essais en tout genre, parfois réalisés par des artistes de renom, comme Edme Bouchardon ${ }^{10}$. En définitive, c'est une Méthode pour apprendre à dessiner réalisée par Charles-Antoine Jombert en 1755, rassemblant tous les modèles les plus utilisés, qui 
connaît un grand succès. Et l'ouvrage ne manque pas de consacrer un chapitre entier à l'étude d'après le modèle vivant, car

en voulant corriger la nature avant que de la connaître parfaitement, on court un grand risque de se maniérer, c'est-à-dire de rendre toujours les choses d'une même façon [...]. La nature, quoique pleine de variétés, a beau leur représenter les choses sous de nouvelles formes, ils ne sont plus en état d'en sentir la différence. [...] C'est pourquoi il faut toujours imiter le modèle avec la plus scrupuleuse exactitude ${ }^{11}$.

C'est pour cette raison que lorsque l'École des élèves protégés est créée en 1749, le premier peintre du roi, Charles-Antoine Coypel, qui la met sur pied, préconise que deux heures par jour soient consacrées à l'étude d'après le modèle vivant ${ }^{12}$.

Avoir l'autorisation de "poser le modèle » constitue un privilège en France jusqu'à la Révolution; il faut en obtenir l'autorisation, ce qui suppose l'affiliation des écoles nouvellement créées et qui se multiplient dans la France du XviIl ${ }^{e}$ siècle, à celle de Paris $^{13}$. La présence ou non d'un cours d'après le modèle vivant permet de distinguer l'ambition de l'école. Celles qui n'en ont pas assurent un enseignement destiné en priorité aux artisans, alors que les écoles académiques et les académies forment des artistes. Sur les quarante-huit écoles d'art ${ }^{14}$ qui fleurissent sur le territoire français, quinze écoles proposent des cours d'après le modèle vivant: Bayonne, Besançon, Bordeaux, Dijon, Grenoble, Lille, Lyon, Marseille, Montpellier, Poitiers, Rouen, Strasbourg, Tours, Toulouse et Valenciennes. Toutes sont affiliées à l'école de Paris, selon le règlement de 1676, même si sept d'entre elles n'en dépendent pas directement pour des raisons liées aux personnalités locales ${ }^{15}$.

Les méthodes et contenus pédagogiques des écoles académiques semblent assez homogènes pendant la période classique, en particulier en ce qui concerne les dessins, estampes et bosses qui sont recommandés bien souvent par l'école parisienne. Cette dernière mène une politique active en matière pédagogique, qui la conduit à envoyer des académies dessinées à Rouen en 1748, à Marseille en $1754^{16}$, à Lyon entre 1721 et 1775, à Tours entre 1785 et $1797^{17}$. Les professeurs des académies de province doivent tenir un registre des pièces qui leur sont confiées $^{18}$. La redondance des éléments patrimoniaux conservés datant du XVIII ${ }^{\mathrm{e}}$ siècle - mêmes manuels de dessins, d'estampes, de moulages - aurait ainsi tendance à accentuer le rôle de la copie dans la pédagogie académique, de même qu'elle suggère qu'une doctrine claire est imposée par la capitale.

Or l'homogénéité s'explique aussi par le fait que les fournisseurs d'estampes ou de bosses sont majoritairement parisiens et que les modèles circulent facilement à la faveur du développement de la gravure " en manière de crayon ", dont le succès est tel auprès des amateurs qu'il s'en imprime une quantité importante, vendue à un prix raisonnable. Le même phénomène s'observe pour les moulages. Une liste des «modèles de bosses » achetés pour l'école de Lille en $1754^{19}$ donne une idée du fonds traditionnel des écoles académiques, qui ne se résume pas du tout à une diffusion du modèle antique : deux figures masculines (Le Fleuve et Pluton), la Vénus et le Mercure de Pigalle, l'Écorché de Bouchardon, quatre têtes séparées, trois jambes en partie séparées, dix pieds séparés, quatre bras séparés et douze trophées en bas-relief.

Le dessin d'après le modèle vivant, unique par essence, est plus aléatoirement conservé dans les institutions dans la mesure où il n'existe pas de " politique de dépôt » explicite, ou, lorsque celle-ci existe, elle n'est pas nécessairement respectée. À l'Académie de Paris, dès la création du «Prix de quartier » en 1684, toutes les œuvres primées sont 
censées être déposées, alors que la conservation des études dessinées d'après le modèle varie en fonction des professeurs et des périodes concernées ${ }^{20}$. Le sort des moulages d'après nature, comme ceux déposés par Le Brun, est encore plus complexe, le matériau étant par essence fragile. Dans les écoles académiques de province, les mêmes aléas entraînent les mêmes conséquences, avec parfois quelques surprises. À Rouen par exemple, alors que l'école a disparu, un fonds de près de deux cents dessins est conservé à la bibliothèque municipale ${ }^{21}$. Le destin de ce type de feuilles, que l'on retrouve fréquemment sur le marché de l'art, dépend aussi de la notoriété de l'artiste ou du fait que les académies dessinées sont recherchées et collectionnées ${ }^{22}$. Il faut aussi noter que les feuilles de nus académiques dessinés ne sont pas non plus forcément un témoignage fiable de cette pratique, dans la mesure où il existe des copies d'après un nu académique dessiné.

Le dessin d'après le modèle vivant demeure au cœur des préoccupations jusqu'à la fin du XVIII ${ }^{e}$ siècle, comme le montre le débat autour du Voltaire nu (1776) de Jean-Baptiste Pigalle. Le sculpteur, qui «désirait depuis longtemps [...] offrir aux jeunes gens un modèle pour l'étude des muscles de l'anatomie dans le goût de l'Écorché de MichelAnge ", se vit proposer de réaliser un monument à la mémoire de Voltaire vivant. « Il y consentit avec plaisir, pourvu qu'il ne fût pas contraint de le vêtir. On l'en laissa le maître. Il exécuta donc le marbre d'après un modèle vivant, le plus laid, le plus décharné et le plus dégoûtant qu'il fût possible de trouver ${ }^{23}$ ". L'" anatomie savante " est le prétexte esthétique, hérité de Michel-Ange, habillant ce vérisme français qui s'incarne dans le modèle vivant.

Le peintre Joseph-Marie Vien dit avoir été le premier à importer la pratique du dessin d'après le modèle vivant dans son atelier privé, très actif dans la seconde moitié du siècle ${ }^{24}$. Celui qui passe pour être le père spirituel du "retour à l'antique» est aussi celui qui perçoit la nécessité du retour à l'observation du vivant, après des années où la correction morphologique pouvait passer pour accessoire chez certains élèves, qui se contentent de dessiner d'après les académies de François Boucher, Carle Vanloo ou Edme Bouchardon, abondamment reproduites dans les diverses méthodes pour apprendre à dessiner. La production massive, dans la deuxième moitié du XVIII ${ }^{\mathrm{e}}$ siècle, du dessin d'illustration exact « d'après nature » contribue à remettre en question cette pratique et à revaloriser l'exactitude. L'Encyclopédie méthodique (1788) s'interroge: «Comment bien voir et comment copier fidèlement si le modèle est mobile ? [...] On a vu des élèves de la première force dans ce genre de dessins faits devant le modèle, n'être pas en état de rendre, je ne dis pas seulement une main ou une tête, mais l'objet le plus simple, avec cette justesse, cette précision en quoi consiste l'art de dessiner exactement ${ }^{25}$.»

\section{Un modèle vivant dessiné « sans maître » : les revendications de la Révolution, la question de l'inventaire du patrimoine des écoles d'art}

20 À l'aube de la Révolution, le journaliste Louis-Sébastien Mercier, favorable aux nouvelles idées, place ce « retour à la nature » comme une nécessité absolue pour tous les arts : "Celui qui, fermant courageusement les livres, pour n'ouvrir que celui de la Nature, ne s'en rapportera qu'à son œil pour voir, à son oreille pour entendre, à son 
cœur pour sentir, saisira le modèle vivant dans son énergique attitude, et frappera ses productions d'un degré de vie que l'autre n'obtiendra point ${ }^{26}$. » La remise en question globale des systèmes académiques va permettre de soulever certains problèmes dans la pratique du dessin d'après le modèle vivant telle qu'elle s'effectue à l'Académie de Paris. L'exercice-roi, qui révèle l'artiste, n'est plus du tout considéré comme une étude permettant d'affirmer son regard personnel et son originalité.

En 1791, le président de la Commune générale des arts (qui remplace les Académies), le peintre Jean-Bernard Restout dénonce une étude du vivant «servile et périodique chaque jour à la même heure, comme ces repas dont l'heure plutôt que l'appétit décide, d'après un modèle toujours le même, présentant toujours les mêmes défauts, défauts que tous copient, et avec lesquels ils se familiarisent chaque jour ${ }^{27}$ ». Antoine Quatremère de Quincy confirme que "de cette redite continuelle de figures sans mouvement, sans caractère et sans expression, résulte ce style privé de vérité, de caractère et d'expression, que les artistes ont désigné eux-mêmes du mot qui en indique la cause : style académique ${ }^{28} »$.

Le dessin d'après le modèle vivant est accusé par le peintre Jacques-Louis David de transmettre un "style académique", avec douze professeurs différents, enseignant "par quartiers" douze "manières" de dessiner ${ }^{29}$. Restout s'en prend aussi à la personne du modèle, fonctionnaire royal, qui sert aussi bien à faire un Jupiter qu'un Mercure. Pire, «tout le monde se rappelle le nommé Deschamps, d'une nature très défectueuse, ayant un large et rouge estomac, des bras minces et des jambes grêles [...] modèle par excellence, copié par tous les artistes, [qui] a tellement rempli leur imagination, qu'il reparaissait sous tous les différents rôles qu'ils lui faisaient jouer dans leurs tableaux ${ }^{30} »$.

En dépit de ces critiques, la Commune des arts échoue à proposer une véritable réforme de l'Académie et du dessin d'après le modèle vivant. Provisoirement, et en l'absence de doctrine élaborée, les artistes engagés dans la Révolution insistent sur une nécessaire étude de l'anatomie et de l'expression afin de sortir des formes plastiques conventionnelles. Il n'est plus question de recourir à une typologie des passions telle qu'elle avait été élaborée au XVII siècle : le rire ou la moue, qui ont pour effet de déformer les traits du visage, sont désormais représentables. Les divers "moqueurs " que le peintre Joseph Ducreux peint pendant la Révolution sont caractéristiques de ces efforts d'expressivité qui veulent s'éloigner de la typologie des "têtes d'expression " fournies traditionnellement comme modèles aux élèves. Enfin, la Société populaire et républicaine des arts, remplaçant en 1793 la Commune des arts, finit par décréter que «l'imitation parfaite de la nature, [est le] seul but de l'Art ${ }^{31}$ ".

24 Fin des modèles donc, et des querelles théoriques. Or, très vite, il apparaît qu'une nature commune ne peut satisfaire le besoin de représentation héroïque de la Révolution. L'expression vraie de l'homme va céder le pas devant l'étude anatomique, conçue comme la base démocratique du dessin, vraie et accessible à tous, permettant de trouver un juste milieu entre l'étude de l'antique et l'observation du modèle vivant, afin de parvenir à ce « beau idéal qu'on doit trouver dans un ouvrage libre ${ }^{32}$ ».

$\mathrm{Au}$ cours de la période révolutionnaire, les académies dessinées transforment profondément la réalité physique du modèle vivant chez nombre d'artistes, acquis ou non à la cause révolutionnaire : les corps masculins deviennent ceux de héros virils, aux muscles gonflés, prêts à incarner l'homme nouveau, à l'image des corps nus qui se déploient dans le grand tableau inachevé de Jacques-Louis David, le Serment du Jeu de 
paume [fig. 4]. Le corps héroïque tend à devenir l'unique mode de représentation de la figure, y compris lorsqu'il s'agit de représenter des femmes, loin du corps plus classique, mais terriblement énigmatique, du deuxième Écorché de Houdon exécuté en 1790.

Figure 4

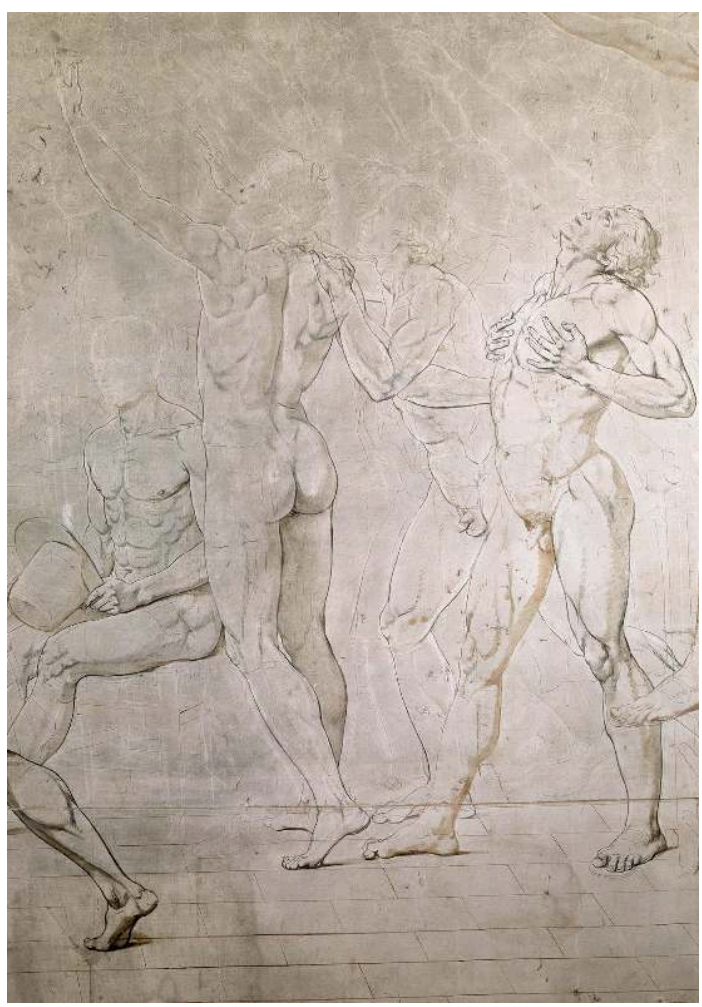

Le Serment du Jeu de Paume (détail), Jacques-Louis David (1748-1825). Dessin (400 × $660 \mathrm{~cm})$

conservé au dépôt du département des Arts graphiques du Musée du Louvre Versailles (inv. MV5841 ; INV26182), musée du château de Versailles et de Trianon.

Reproduction RMN-Grand Palais.

Le corps vivant, en dépit de la mise en place au sein de l'école parisienne de cours de dissection, semble ainsi à la fois être au cœur de l'enseignement et paradoxalement, plus que jamais retravaillé, même si la présence d'un modèle demeure la référence pour choisir un atelier, et fonde le succès de celui de David ${ }^{33}$. Un dessin aquarellé de l'atelier de Jean-Baptiste Regnault, grand atelier rival de Jacques-Louis David, montre cependant un modèle vivant de femme perdu au milieu de quantité de moulages en grandeur naturelle [fig.5]. L'existence d'un atelier public de moulage à vocation pédagogique en France à partir de la Convention, garant de la fidélité des plâtres aux originaux, va contribuer à relancer la pratique de la copie ${ }^{34}$. 
Figure 5

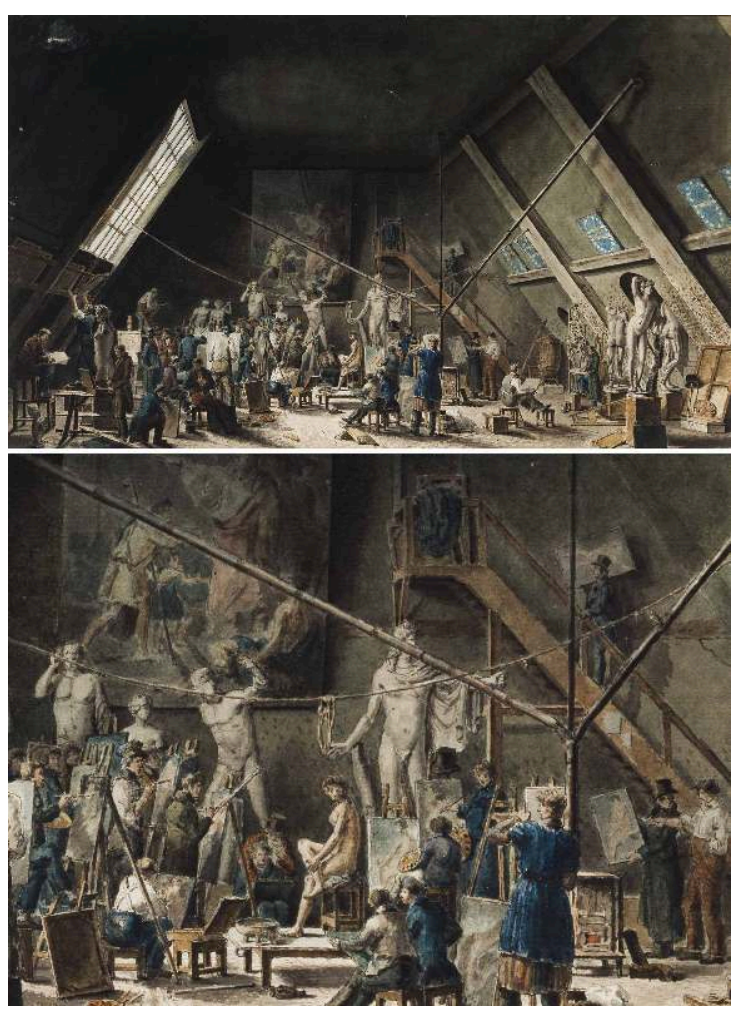

Intérieur de l'atelier de M. Regnault, dessinateur inconnu, vers 1800. Dessin à la plume et lavis à l'encre de Chine, aquarelle $(30$ × $50 \mathrm{~cm}$ ), conservé à la Bibliothèque nationale de France (Réserve FOL-VE-53 D), Paris.

Reproduction BnF.

27 Il existe bien une tension pendant la période révolutionnaire, un goût pour la vérité et un besoin d'un corps héroïque. Au sein de l'école parisienne, on dissèque depuis 1795 et au moins jusqu'en 1798, comme l'atteste la présence d'une table de dissection au milieu des moulages antiques ou anatomiques ${ }^{35}$... mais si le vivant est sur la table, les muscles de Michel-Ange sont sur la toile du Serment du Jeu de paume... Que reste-t-il dans les écoles d'art comme témoignage de cette période de bouleversements? Quelques dessins du corps disséqué, destinés ou non à un ouvrage, de Jacques Réattu à JeanBaptiste $W_{i c a r}^{36}$, et conservés dans des institutions muséales alors qu'ils furent probablement réalisés au sein de l'Académie de France à Rome, foyer de l'effervescence révolutionnaire française en Italie [fig. 6]. 
Figure 6

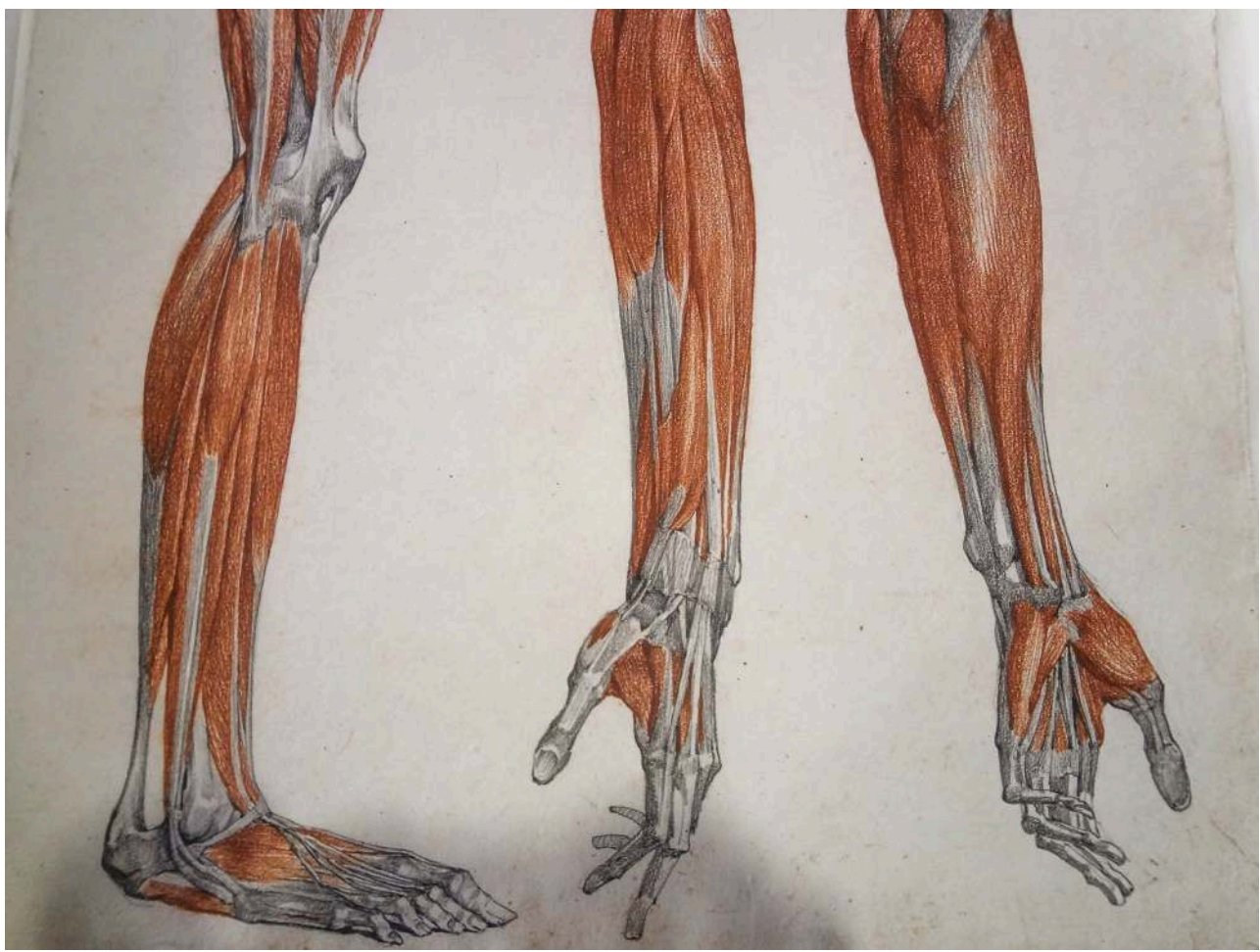

Étude anatomique de deux bras et d'une jambe, Jacques Réattu (1760-1833), dessin à la sanguine et à la pierre noire (59,5 x $44 \mathrm{~cm}$ ) conservée au musée Réattu (inv. 868.1.664), Arles.

Reproduction Morwena Joly-Parvex.

Le point le plus important au regard du patrimoine des écoles d'art concerne la prise de conscience de la valeur patrimoniale des modèles et œuvres de réception. D'une manière générale, les premiers inventaires des écoles d'art, plus particulièrement des écoles académiques, sont dressés pendant la période révolutionnaire, érigeant ce matériel pédagogique en patrimoine de la nation. À Rouen par exemple, c'est un inventaire réalisé sous la Révolution qui nous permet de savoir quels étaient les modèles de l'école académique: un Apollon et Daphné, le Milon de Falconet et celui de Puget, Vénus et Mercure de Pigalle, l'Écorché de Houdon, Le Bacchus, le Gladiateur, la Vénus Médicis, la Vénus pudica, des têtes d'enfant par Jacques-François Saly ou François Lucas, le Laocoon, une tête du Temps et une tête de femme représentant le Commerce (par Paul-Ambroise Slodtz pour le Méridien de la Bourse du commerce de Rouen), nombre de morceaux en terre cuite ${ }^{37}$. Liste qui témoigne au passage de l'équilibre entre les modèles modernes et antiques proposés à la copie.

Le débat autour du degré d'idéalisation nécessaire au dessin du corps vivant s'atténue au début du XIX ${ }^{e}$ siècle à la faveur de la diffusion de la doctrine esthétique héritée de Jacques-Louis David par son "école", en réalité très diverse dans ses buts et ses pratiques. Si ses préceptes sont diffusés largement dans les écoles d'art françaises, c'est qu'ils dépassent le débat qui opposait les partisans du «Beau réel » et ceux du «Beau idéal ». Cependant, les principes davidiens, à savoir une nature fidèlement observée mais corrigée par l'antique, s'affadissent tout au long du XIX ${ }^{e}$ siècle. Lignes idéalisées et notations réalistes individuelles vont coexister au sein des œuvres, dans l'oubli de la synthèse propre à David. Ce "formalisme académique ", constamment dénoncé par les amateurs de la deuxième moitié du XIX ${ }^{e}$ siècle, culmine paradoxalement à un moment 
où l'École des beaux-arts de Paris n'est plus vraiment structurée par une théorie esthétique claire.

L'exercice-roi demeure le dessin d'après le modèle vivant, où l'outrance du corps viril révolutionnaire décroît rapidement au profit d'une simplification de la ligne et d'un poli de la surface. Un des élèves de David, le peintre Jean-Dominique Ingres, qui forme près de quatre cents élèves dans son atelier, prend très vite ses distances avec l'observation du modèle vivant. L'étude dessinée doit en effet être retravaillée pour l'exécution du tableau, car «si l'on peint les personnages du tableau en copiant directement le modèle, la peinture absorbe le prosaïsme de la réalité ${ }^{38} »$. Hippolyte Flandrin, l'élève préféré du maître, puis son disciple Jean-Baptiste Poncet, assurent la postérité des préceptes d'Ingres [fig. 7].

Figure 7

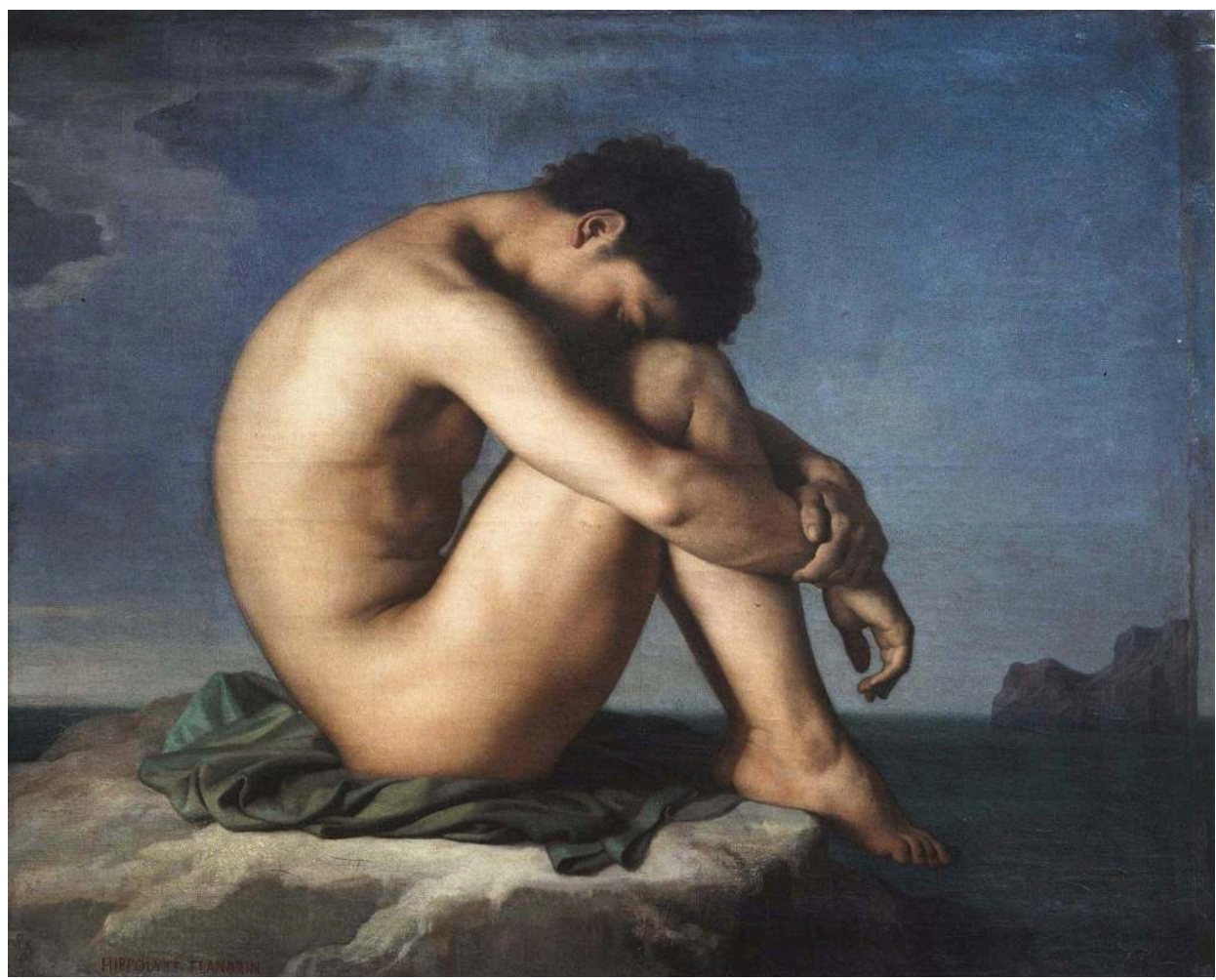

Jeune homme nu assis au bord de la mer, Hippolyte Flandrin (1809-1864), 1836. Huile sur toile (98 x $124 \mathrm{~cm}$ ) conservée au Musée du Louvre (inv. MI171), Paris.

Reproduction C2RMF.

L'étude anatomique et morphologique est de plus en plus séparée du projet artistique, qui s'effectue au sein des ateliers de peinture et de sculpture, en dehors des écoles académiques jusqu'en 1863. Au sein du célèbre atelier du peintre William Bouguereau, le thème privilégié est celui de la femme nue, parfaitement idéalisée. Entrant dans l'atelier du peintre en 1891, Henri Matisse évoque ainsi «la "perfection" des figures peintes qu'on y fabriquait du lundi au samedi et tellement insignifiantes que cette "perfection" vide me donnait le vertige $\mathrm{e}^{39}$ ".

Dans la deuxième moitié du XIX ${ }^{\mathrm{e}}$ siècle, à la faveur des créations du mouleur Alexandre Desachy $^{40}$, de nombreux modèles antiques en plâtre vont être acquis par les écoles d'art partout en France, formant un «musée imaginaire » accessible à tous et sur tout le 
territoire. Comme dans toute l'Europe ${ }^{41}$, les moulages ont connu un véritable âge d'or au XIX siècle, essaimant d'innombrables petits "musées de plâtres ", le plus souvent gréco-romains, pouvant laisser penser qu'une véritable hégémonie du modèle antique s'impose dans la pédagogie, à l'image du « musée des études » créé en 1832 à l'école de Paris $^{42}$. Quantité de dessins ou de bosses d'après ces modèles sont bien conservés pour témoigner de cette intense activité de copie, qui fait l'objet de prix d'émulation. Certes, le fait que l'univers visuel d'artistes en formation soit saturé quotidiennement de telles références n'est pas anodin, mais il n'est pas non plus sans produire son effet inverse : un fiévreux besoin de modèles vivants [fig. 8].

Figure 8

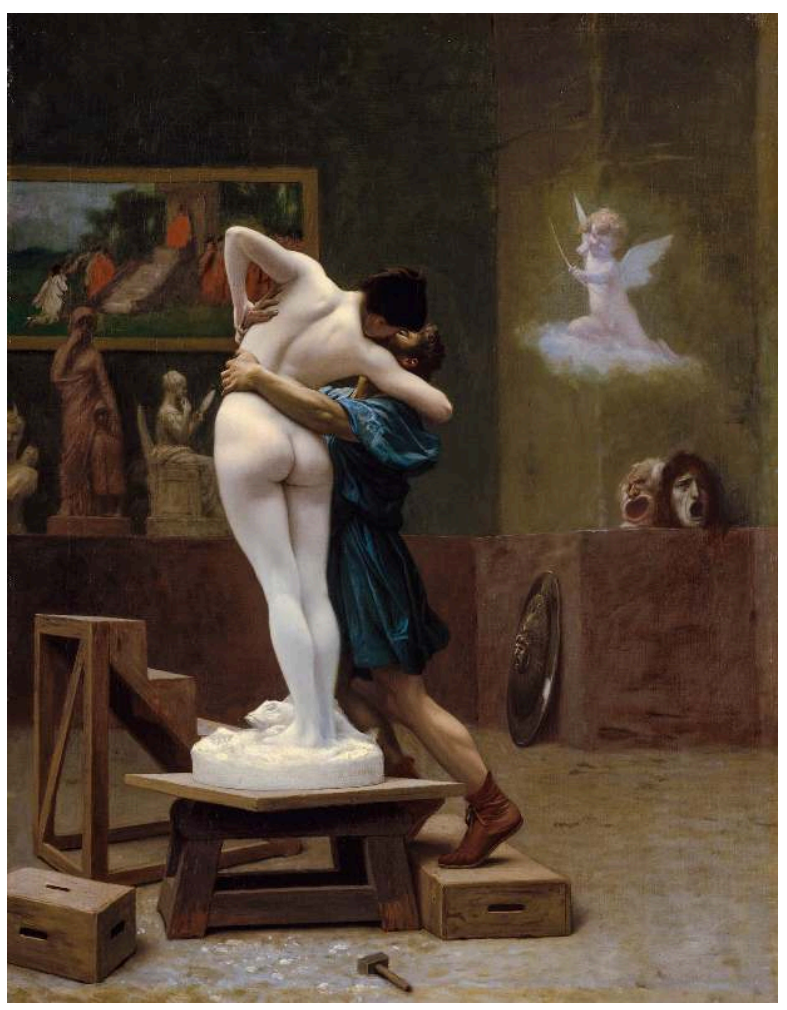

Un moulage sur nature chez Haviland à Auteuil, Édouard Dantan (1848-1897), 1887. Huile sur toile (165 x $131,5 \mathrm{~cm}$ ) conservée au Musée des beaux-arts de Göteborg (inv. F 21), Suède.

Reproduction Musée des beaux-arts de Göteborg.

Deux œuvres littéraires témoignent de l'obsession des artistes pour l'imitation du corps vivant, qu'explicite Louis-Léon Gérôme avec son Pygmalion et Galatée (1890). Le peintre Claude Lantin, héros de L'CEuvre d'Émile Zola (1886), s'interroge : « Très épatant tout de même le nu [...]. Et ça vibre et ça prend une sacrée vie, comme si l'on voyait le sang couler dans les muscles ${ }^{43}$. " Le vieux peintre Frenhofer, imaginé par Honoré de Balzac dès 1831, comprend lui aussi que le "poli » des carnations hérité d'Ingres rend les corps froids comme le marbre: "Le sang ne court pas sous cette peau d'ivoire, l'existence ne gonfle pas de sa rosée pourpre les veines fibrilles qui s'entrelacent sous la transparence ambrée des tempes et de la poitrine ${ }^{44}$. " Cette fascination pour le corps vivant du modèle s'incarne avec Édouard Manet et son fameux modèle Victorine Meurent ${ }^{45}$, aux formes massives et à la sensualité lourde, célébrée en 1863 dans le Déjeuner sur l'herbe et l'Olympia. Les commentaires contemporains et postérieurs ne 
manquent pas de se demander si le sujet de la toile n'est pas aussi la relation du peintre au modèle vivant.

Significativement, la grande réforme opérée en 1863 à l'École des beaux-arts de Paris ne change rien à l'organisation de l'exercice tel qu'il se présente depuis le XVII siècle. L'étude du nu à partir du modèle vivant revêt une telle importance dans la formation française que les écoles d'art privées qui naissent à Paris au XIX ${ }^{\mathrm{e}}$ siècle fondent leur succès sur cette pratique désormais libre. En 1815 naît ainsi l'académie Suisse, fondée par le genevois Charles Suisse, un ancien modèle de Jacques-Louis David. Plus tard, en 1868, le peintre Rodolphe Julian fonde un atelier avec une étude du modèle vivant où les femmes sont acceptées. Cette école acquiert dans ce domaine une célébrité internationale au point de contrôler plusieurs ateliers des deux côtés de la Seine ${ }^{46}$.

Figure 9

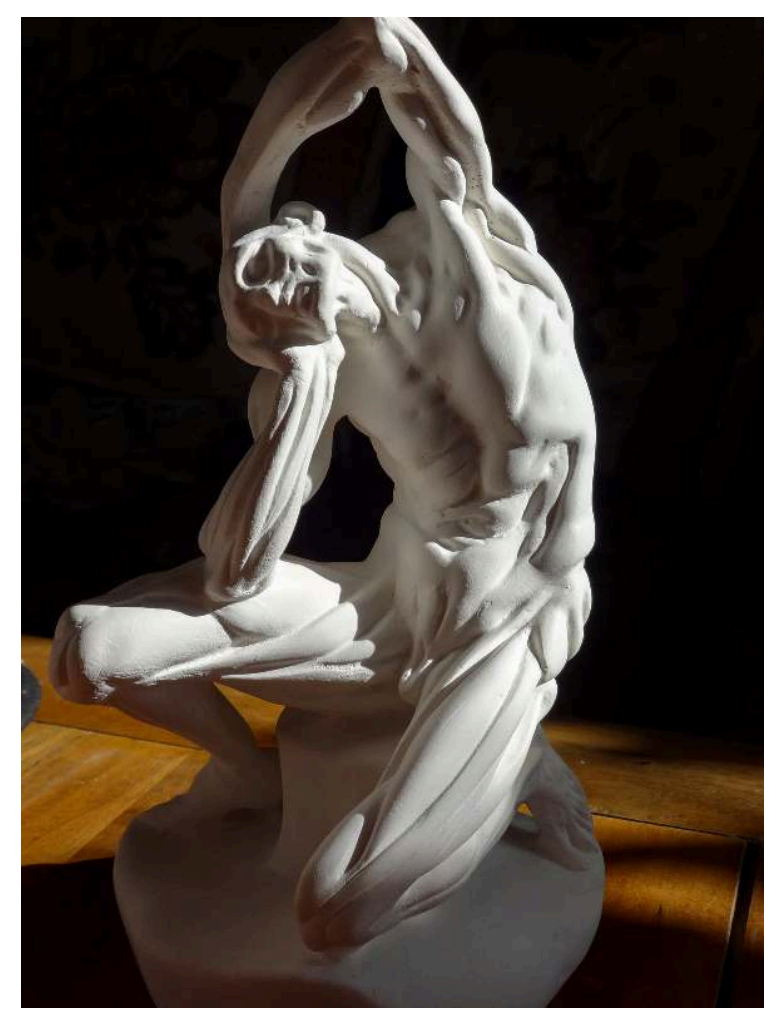

Écorché dit de Michel-Ange, XIXe siècle, moulage contemporain $(25 \times 12,5 \times 5 \mathrm{~cm})$ conservée dans une collection particulière.

Reproduction Morwena Joly-Parvex.

Si l'enseignement à l'École des beaux-arts de Paris est dominé par les élèves de David, la carrière de peintres nettement déclarés en faveur d'une observation réaliste de la nature n'est en rien empêchée et rencontre même les faveurs officielles. Cette "résistance réaliste» prend appui sur l'étude du corps disséqué, qui se comprend comme volonté de percer les mystères du corps réel. Théodore Géricault ou Eugène Delacroix multiplient les dessins d'après des traités d'anatomie ou de l'Écorché attribué à Michel-Ange [fig. 9]. Delacroix va même jusqu'à réaliser un nombre important de dessins d'après dissection, poussant l'étude du réel jusqu'à un point radical, non loin d'une forme de nihilisme pédagogique ${ }^{47}$. À cette même époque, la pratique de la dissection s'installe au cœur de l'École des beaux-arts de Paris ${ }^{48}$. Ainsi, au sein même de 
l'institution se développe un enseignement qui se place à rebours de ce qui est transmis dans les grands ateliers des peintres et des sculpteurs de tradition néoclassique. De cette époque ne restent que peu de traces, hormis quelques dessins myologiques de Delacroix [fig. 10]. Dans les écoles d'art en région, des cours d'anatomie, qui étaient déjà dispensés dans les écoles académiques, continuent vraisemblablement à l'être, mais aucune trace matérielle ne vient pour l'heure confirmer cette pratique pédagogique. À Angers cependant, le musée des Beaux-Arts conserve une impressionnante série de dessins anatomiques exécutés par le sculpteur David d'Angers qui atteste d'une activité dans ce domaine pratiquée dans certaines régions.

\section{Figure 10}

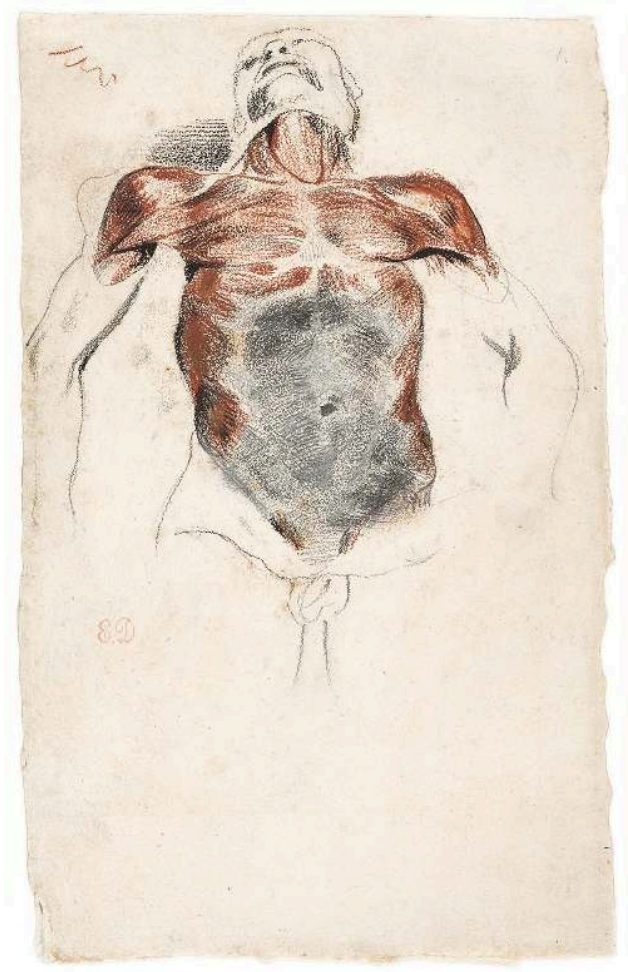

Torse écorché, Eugène Delacroix (1798-1863), vers 1828. Dessin à la pierre noire, rehaussé de sanguine et de craie blanche $(25,2 \times 15,9 \mathrm{~cm})$ conservé au Metropolitan Museum of Art (inv. 2013.1135.23), New York.

Reproduction Metropolitan Museum of Art.

Cet attrait marqué pour le dessin d'après le cadavre rappelle la volonté héritée de la Renaissance de fouiller la nature afin de comprendre son essence, comme chez Léonard de Vinci, Michel-Ange ou Dürer. Imiter la nature n'est-il pas le seul et puissant horizon esthétique de l'artiste, célébrant et voulant rivaliser avec le vivant?

Le $\mathrm{XIX}^{\mathrm{e}}$ siècle revivifie progressivement le débat du siècle précédent, entre «Beau idéal » et « Beau réel », même si la réflexion se raidit, tant la « copie » et la " tradition » ont été portées par les grands maîtres de l'école française comme Ingres et ses élèves. Quelle place accorder désormais à l'étude du modèle vivant à l'heure où son succès dans les académies privées s'affirme ? Et surtout, à l'heure où les artistes émergents, qui ne passent pas par l'École des beaux-arts, structurent une offensive esthétique contre l'idéalisme. Gustave Courbet va jusqu'à affirmer que « le beau donné par la nature est supérieur à toutes les constructions de l'artiste ${ }^{49}$ ». Son célèbre Atelier du peintre (1855), 
qui, refusé au Salon, lui fait instituer un Salon du réalisme concurrent, est lui aussi explicite quant à la place du modèle vivant : la figure nue est placée comme un centre de gravité au milieu du tableau, alors que le peintre esquisse un paysage. Le critique Gustave Planche entrevoit très clairement les enjeux esthétiques forts qui se nouent autour de l'étude du modèle vivant et de la tradition: "Je comprends très bien le danger de la tradition séparée de l'étude assidue de la nature, mais je comprends également comme une vérité évidente le danger d'une étude exclusive de la nature ${ }^{50}$. "

La résistance à l'idéalisme esthétique dans la pratique du dessin d'après le modèle vivant s'est longtemps incarnée par la référence à la statuette écorchée attribuée à Michel-Ange, jusque tard dans le XIX ${ }^{e}$ siècle. Une ronde-bosse qui témoigne du réel du corps et de ses formes complexes, dont les moulages en plâtre se répandent dans tous les ateliers d'artistes. Les moulages écorchés fréquemment conservés dans les fonds patrimoniaux des écoles d'art - celui attribué à Michel-Ange ou celui attribué à Bandinelli, ceux de Houdon -, matérialisant cette « résistance réaliste » qui s'appuie sur le dessin d'après nature, loin de tous les modèles proposés à la copie, doivent être davantage mis en valeur au sein du patrimoine pédagogique. Lorsque Gustave Courbet conçoit un de ses premiers autoportraits, l'artiste fait figurer à ses côtés la fameuse statuette écorchée $e^{51}$. Paul Cézanne, Gustave Moreau ${ }^{52}$ ou Henri Matisse puisent dans cette " grammaire " du corps les principes mêmes de la simplification des formes qui sert désormais à saisir le vivant du corps, cette matière insaisissable qui demeure le sujet de l'artiste.

39 Fait paradoxal, alors que la photographie pourrait utilement être utilisée dans le travail de la création, fixant un temps le réel du corps, cette dernière ne remplace aucunement la présence physique du modèle. De fait, la photographie de modèles vivants est bien présente dans les fonds patrimoniaux des écoles d'art, de même que les séances de pose d'après le modèle, mais trop peu de textes viennent documenter l'usage de tels clichés dans la pratique pédagogique, le réel de la chair échappant largement à la photographie et son point de vue unique [fig. 11]. 
Figure 11

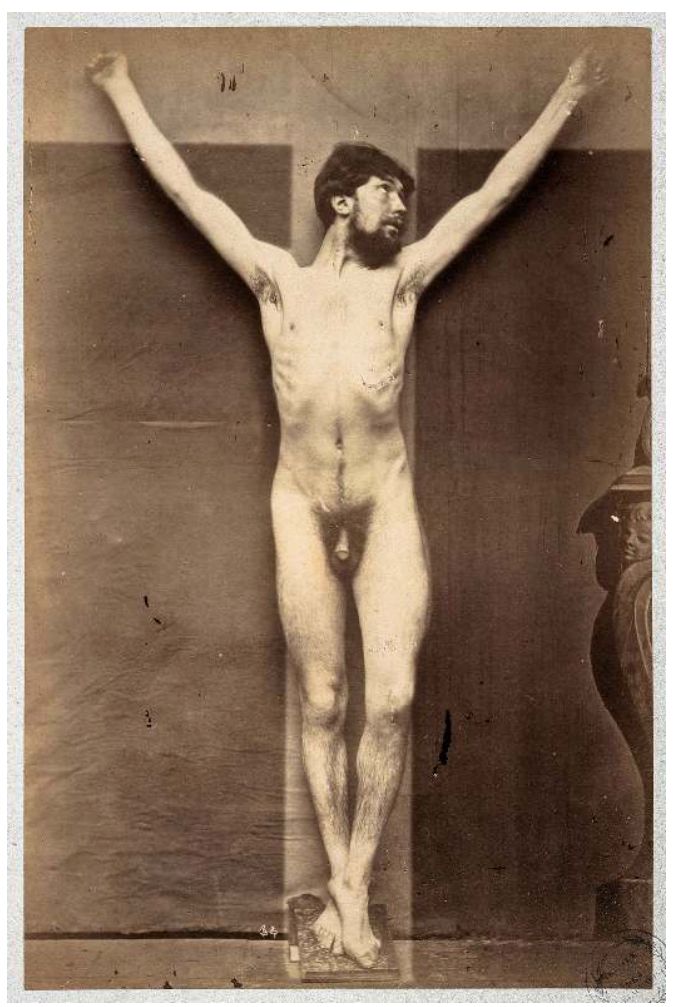

Modèle masculin nu en croix, Gaudenzio Marconi (1841-1885), photographie conservée à l'École nationale des beaux-Arts de Paris.

Reproduction RMN-Grand Palais.

\section{Le tournant fin de siècle et le $\mathrm{xx}^{\mathrm{e}}$ siècle : l'érotique du corps vivant au cœur de l'énergie créatrice}

Si le public est désormais prêt à apprécier et même rechercher une peinture qui n'est plus centrée sur la mimêsis depuis les impressionnistes et symbolistes, et si l'œuvre d'art est de plus en plus comprise comme une vision singulière du réel, il n'en demeure pas moins qu'une émotion partagée avec l'artiste et son imaginaire est encore facilitée par les traces d'une figuration du corps. L'étude d'après modèle vivant s'impose au-delà des écoles d'art, et sa pratique continue dans les ateliers privés d'artistes, d'autant que nombre de femmes acceptent de devenir modèles. Même Paul Cézanne, connu pour être mal à l'aise avec cet exercice lorsqu'il suit les cours de l'académie Suisse finit par l'adopter, au grand étonnement du sculpteur Jules Gibert qui lui enseignait le dessin d'après le modèle vivant ${ }^{53}$. Ce dernier lui rend visite à Paris et se voit ouvrir la porte «par une femme complètement nue, qui me fit entrer dans l'atelier où Cézanne peignait $^{54} »$.

Le singulier parcours de Marcel Duchamp au regard de l'étude d'après le modèle vivant mérite d'être souligné, tant le corps réel semble avoir fasciné l'inventeur des readymade. Il s'inscrit à l'académie Julian en 1904 afin de préparer le concours l'École des beaux-arts de Paris : «Le premier examen consistait en un dessin d'après le nu, au fusain ; j'ai été recalé55. » Son mépris affiché par la suite pour la formation académique, et en particulier pour le dessin d'après le modèle vivant, s'exprime sans doute avec son 
$\mathrm{Nu}$ descendant l'escalier $n^{\circ} 2$ (1911-1912) : "Peint comme il est, en sévères couleurs de bois, le nu anatomique n'existe pas, ou du moins, ne peut pas être vu car je renonçai complètement à l'apparence naturaliste d'un $\mathrm{nu}^{56}$.» Et pourtant, son ultime œuvre réalisée au long de vingt années (de 1946 à 1966), place la matérialité de la chair au cœur de sa recherche obsessionnelle. Étant donné..., installation voyeuriste cruelle d'un corps de femme, est l'objet de multiples tentatives matérielles. Marcel Duchamp réalise d'abord une version du corps avec du cuir sur relief de plâtre, puis achète des seins postiches en caoutchouc mousse. Son souhait est de trouver "une couleur de peau aussi naturelle que possible [qui] n'a plus rien à faire avec le modèle en plastiline que j'ai fait mouler. [...] Avec la peau, je me prépare de beaux jours de difficulté57 ». Le 3 octobre 1968, à la mort de Marcel Duchamp, l'œuvre est enfin découverte dans son atelier clandestin : "Une vision qui vous cloue [...]. La figure était recouverte de peau de porc qui se desséchait ${ }^{58}$. Corps vivant ou mort, c'est bien la matière érotique de la relation avec le vivant qui se manifeste comme étant au cœur des dernières recherches de Marcel Duchamp, écho singulier à ses premières relations d'artiste avec le nu posé à l'académie Julian ou au concours de l'École des beaux-arts [fig. 12, fig. 13].

Figure 12

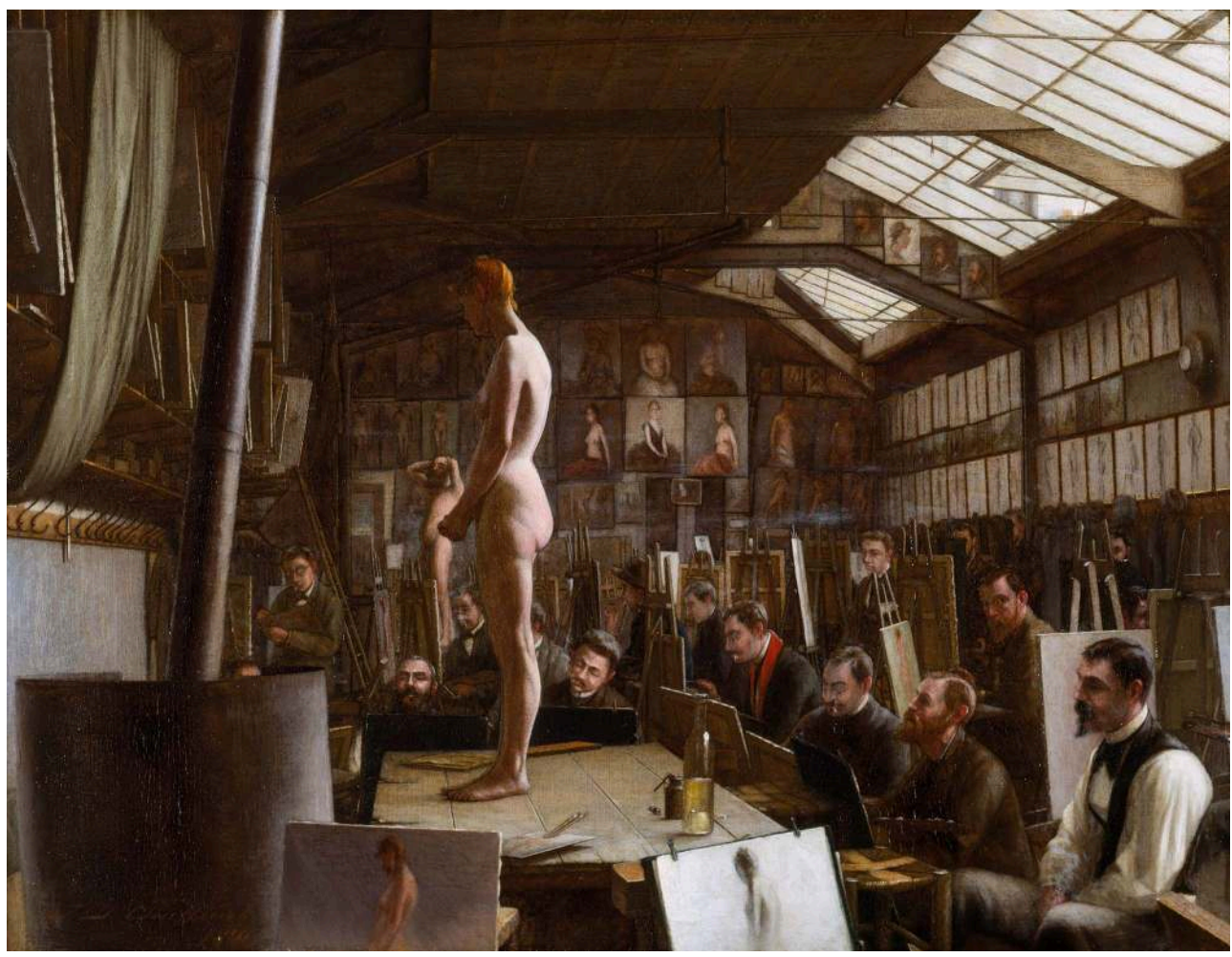

L'Atelier de Bouguereau à l'Académie Julian, Jefferson David Chalfant (1856-1931), 1891. Huile sur panneau de bois (28,5 × 36,8 cm) conservée au San Francisco De Young Museum (inv. 1979.7.26), San Francisco.

Reproduction San Francisco De Young Museum. 
Figure 13

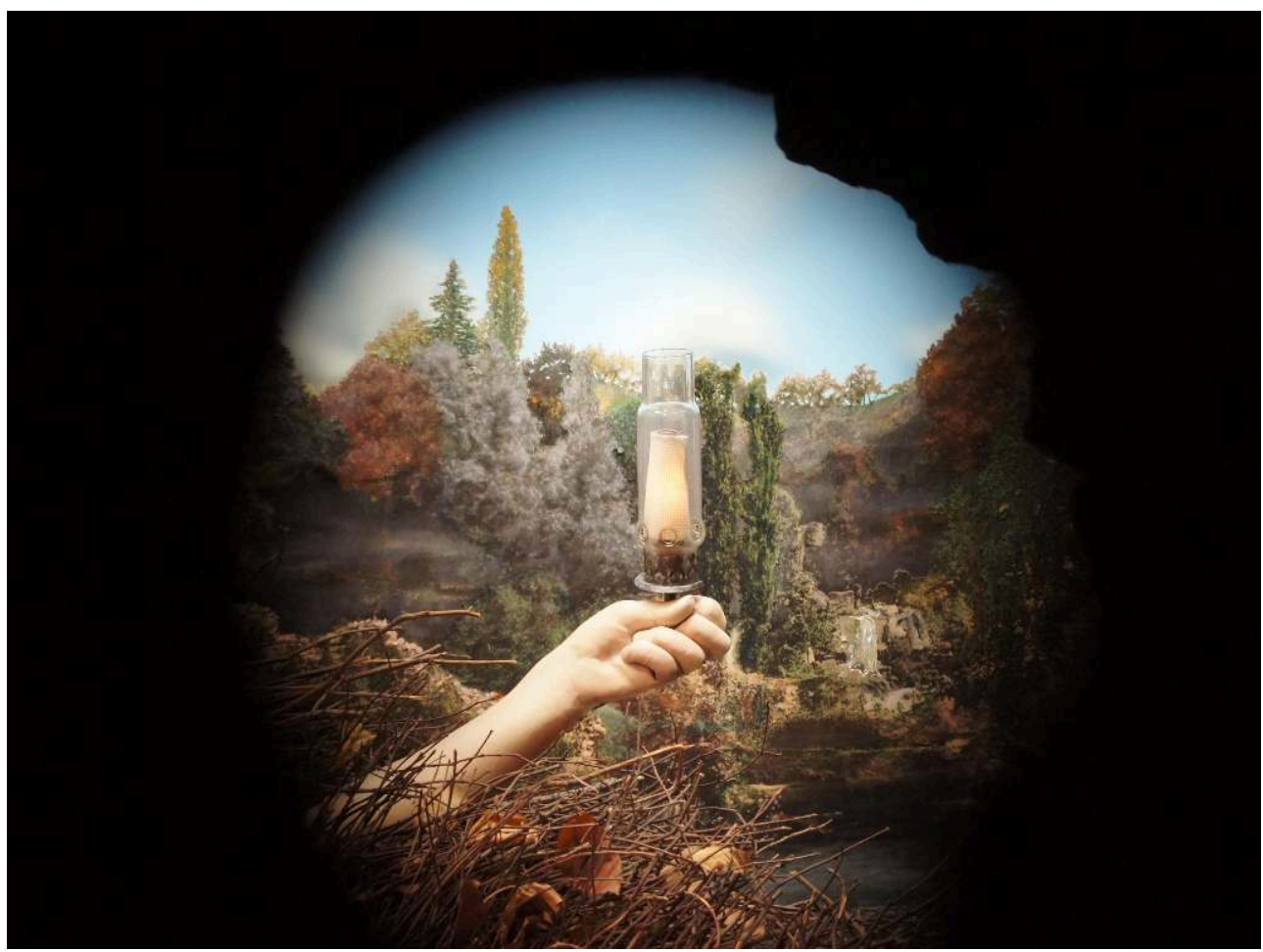

Étant donné : $7^{\circ}$ la chute d'eau, $2^{\circ}$ le gaz d'éclairage, Marcel Duchamp (1887-1968), 1946-1966, vue de l'intérieur de l'œuvre. Assemblage de matériaux mixtes $(242,6$ × 177,8 x 124,5 cm) conservée au Philadelphia Museum of Art (inv. 1969-41-1), Philadelphie.

Reproduction Morwena Joly-Parvex.

$\mathrm{Au}$ terme de cette vue cavalière sur la place de l'étude d'après modèle vivant dans la pédagogie, puis dans l'imaginaire de l'artiste, mettant au jour la tension érotique entre les corps au cœur du processus créateur, il reste à parcourir la deuxième moitié du $\mathrm{xx}^{\mathrm{e}}$ siècle. Paul Landowski, artiste et professeur à l'École des beaux-arts, s'interroge en 1943 sur l'essence de la pédagogie de l'École des beaux-arts. Son ouvrage s'achève sur l'importance de l'étude d'après le modèle vivant, marquant « cette poussée interne du nu, qui n'est pas autre chose que la force de la réalité refusant de se laisser éliminer de l'Art $^{59}$ \%. Le modèle antique est clairement mis de côté dès la Deuxième Guerre mondiale, de même que tous les plâtres accumulés au XIX ${ }^{e}$ siècle dans toutes les écoles d'art, dont l'intérêt ne réside plus désormais que dans l'initiation "aux procédés techniques de mise en place ». « Le meilleur guide pour comprendre les formes vivantes est l'anatomie ${ }^{60} "$, dont les écorchés sont les symboles. Ces éléments patrimoniaux demeurent les seules traces tangibles de l'importance du modèle vivant dans la pédagogie.

Lorsque Henri Matisse opère une révolution dans la représentation du corps vivant avec sa série de Nus bleus composés à l'aide de papiers découpés, dans les années cinquante, le sens de ce renouvellement des formes corporelles échappe en grande partie si on n'y reconnaît pas une évocation de l'Écorché attribué à Michel-Ange. Henri Matisse, qui avait exécuté un écorché en bronze en $1903^{61}$ qui lui était très semblable, reprend sa pose dans sa série de nus bleus aux attitudes tourmentées. Version singulière et intense, où le simple jeu des pleins et des vides fixe la présence symbolique du corps. La figurine d'atelier en plâtre symbolise ce réel du corps qui 
fascine Henri Matisse, car ils appartiennent au même imaginaire du vivant, la dimension érotique en moins. "Le modèle, pour les autres, c'est un renseignement, moi, c'est quelque chose qui m'arrête. C'est le foyer de mon énergie [...]. Je dessine tout près du modèle - en lui-même - les yeux à moins d'un mètre du modèle et le genou pouvant toucher le genou ${ }^{62} »[$ fig. 14].

Figure 14

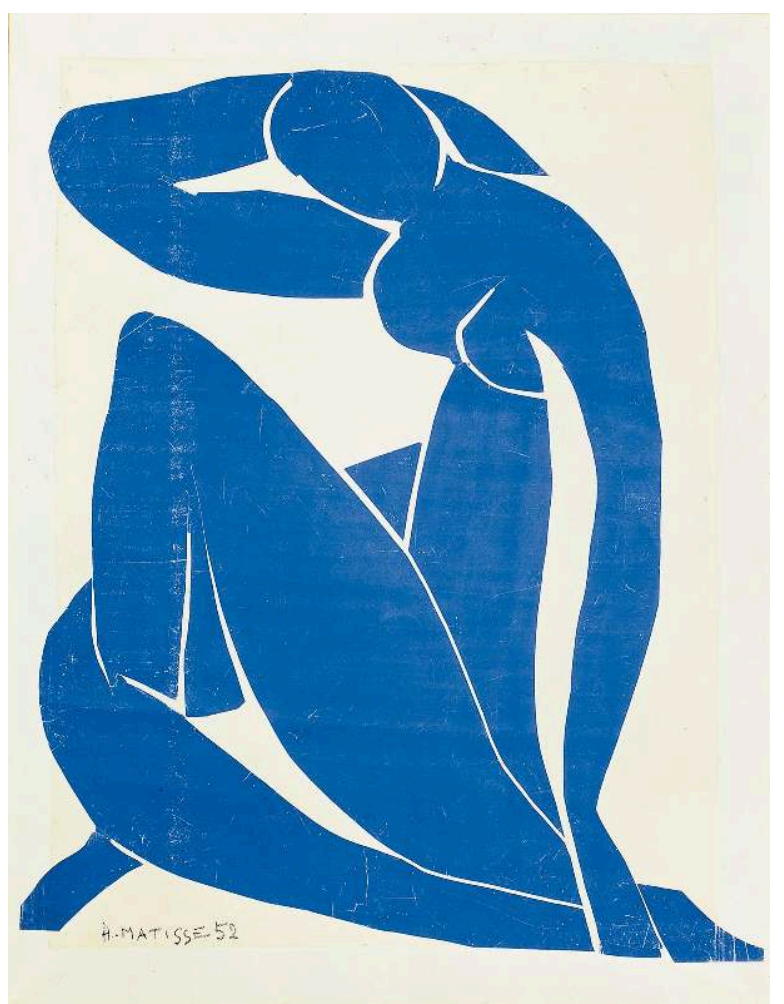

Nu bleu II, Henri Matisse (1869-1954), 1952. Papiers gouachés, découpés et collés sur papier marouflé sur toile (103,8 x $86 \mathrm{~cm})$ conservés au Centre Pompidou - Musée national d'art moderne - Centre de création industrielle (inv. AM 1984-276), Paris.

(C) Succession Henri Matisse / reproduction Centre Pompidou, MNAM-CCl, distribution RMN-Grand Palais.

Les écoles d'art de France n'ont pas cessé de proposer ce face-à-face crucial entre l'artiste et la nature. Si les réformes engagées à la suite du mouvement contestataire de 1968 à l'École nationale supérieure des beaux-arts n'ont eu que peu de conséquences sur le cours d'après le modèle vivant, le terme de morphologie lui est désormais préféré. Peu de choses étaient en réalité à changer dans cet exercice, le dessin du corps n'ayant jamais réussi à être véritablement normé par l'institution académique qui, du reste, ne l'a jamais véritablement souhaité. Au-delà de tous les modèles proposés, c'est la voie singulière de l'artiste qui a toujours été cherchée à travers cet exercice.

Le grand invariant demeure cette fascination pour la matérialité du corps avec Yves Klein et ses Anthropométries exécutées à partir de 1958, où le modèle vivant devient luimême le pinceau. Le geste d'Yves Klein, qui n'est en rien unique, aura une importante postérité avec le courant du body art, initié par Dennis Oppenheim, Bruce Nauman et Vito Acconci à partir de $1969^{63}$.

Comment le patrimoine des écoles d'art pourrait-il se passer de cette réflexion dans sa volonté de transmettre une histoire sensible de la pédagogie artistique? Au milieu de la 
masse des modèles en plâtre patinés par le temps, des estampes "pour apprendre à dessiner", des albums photographiques de modèles témoignent certes tous de la formation dispensée et de l'effort de la réflexion pédagogique. Seuls les dessins d'après le modèle vivant ou les académies peintes, en nombre suffisant pour être comparés, pourraient être à même de rendre compte de la confrontation au corps vivant, si essentielle à l'artiste en ce qu'elle permet de comprendre sa propre singularité, mieux que l'objet ou le paysage, dans la mesure où le corps est l'intime par excellence. Le corps est demeuré, au fil des siècles, le point imaginaire par lequel l'artiste doit passer pour avoir accès à sa propre intelligibilité.

\section{NOTES}

1. Soit quinze écoles (voir infra). Pour l'enseignement délivré dans les autres écoles d'art, voir ENFERT Renaud d', L'Enseignement du dessin en France. Figure humaine et dessin géométrique (1750-1850), Paris, Belin, 2003.

2. L'Académie de France à Rome dispose de ce même privilège.

3. « Article IV. L'Académie sera ouverte tous les jours de la semaine, excepté les dimanches et les fêtes, à la jeunesse et aux étudiants, pour y dessiner l'espace de deux heures, et profiter des leçons qu'on fera sur le modèle qui sera mis en attitude par le professeur, comme aussi pour apprendre la géométrie, la perspective et l'anatomie [...] les professeurs serviront chacun un mois de l'année, et se trouveront tous les jours à l'heure prescrite pour faire [...], poser le modèle, le dessiner ou modeler, afin qu'il serve d'exemple aux étudiants. »

4. ANTOINE Annie \& MICHON Cédric (dir.), Les Sociétés au XVII siècle. Angleterre, Espagne, France, Rennes, Presses universitaires de Rennes, coll. « Histoire ", 2006, p. 344.

5. GUIFFREY Jean, MARCEL Pierre \& ROUCHÈS Gabriel, Inventaire général des dessins du Musée du Louvre et du Musée de Versailles. École française, tome 3, Callot-Corneille, Paris, Librairie centrale d'art et d'architecture, 1909, p. x.

6. TESTELIN Henri, Mémoires pour servir à l'histoire de l'Académie royale de peinture et de sculpture, Paris, P. Jannet, coll. « Bibliothèque elzévirienne », 1853, p. 56-57.

7. JOLY Morwena, «Le modèle antique examiné sous l'angle anatomique : entre beau idéal et beau réel », Dix-huitième siècle, 2009, n 41, vol.1, «individus et communautés », p. 393-408 [disponible en ligne], https://www.cairn.info/revue-dix-huitieme-siecle-2009-1-page-393.htm [lien valide en janvier 2021].

8. DIDEROT Denis, Salon de 1765. Essais sur la Peinture. Beaux-arts I, Paris, Hermann, 1984 [1765], p. 18.

9. DIDEROT Denis \& ALEMBERT Jean d' (dir.), Encyclopédie ou Dictionnaire raisonné des sciences, des arts et des métiers, tome 4, article « dessin », Paris, Briasson, 1751-1772, p. 890.

10. JOLY Morwena, La Leçon d'anatomie. Le corps des artistes de la Renaissance au romantisme, Paris, Hazan, 2008, p. 77-133.

11. JOMBERT Charles-Antoine, Méthode pour apprendre le dessein..., Paris, Imprimerie Jombert, 1755, p. 50.

12. TISSIER Jacqueline-Edmonde, «Félix Lecomte (1737-1817)», in RABREAU Daniel (dir.), Paris, capitale des arts sous Louis XV. Peinture, sculpture, architecture, fêtes, iconographie, Bordeaux, Art \& arts, coll. « Annales du Centre Ledoux », 1997, p. 131. 
13. PHILOUZE-LAHALLE Agnès, Les Écoles de dessin au XVIII ${ }^{e}$ siècle. Entre arts libéraux et arts mécaniques, Rennes, Presses universitaires de Rennes, coll. « Histoire », 2006, p. 94.

14. PHILOUZE-LAHALLE Agnès, Les Écoles de dessin au XVIII ${ }^{e}$ siècle, p. 210.

15. Rouen, Lyon, Dijon, Lille, Strasbourg, Besançon et Toulouse.

16. VI-TONG Nelly, «

17. BRUGEROLLES Emmanuelle (dir.), L'Académie mise à nu. L'École du modèle à l'Académie royale, catalogue d'exposition (École nationale supérieure des beaux-arts, Paris, 26 octobre 2009-29 janvier 2010), Paris, Beaux-arts de Paris les éditions, coll. « Carnets d'études », 2009, p. 32-34.

18. PHILOUZE-LAHALLE Agnès, Les Écoles de dessin au XVIII ${ }^{e}$ siècle, p. 228.

19. PHILOUZE-LAHALLE Agnès, Les Écoles de dessin au XVIII siècle, p. 228-229.

20. BRUGEROLLES Emmanuelle (dir.), L'Académie mise à nu, p. 30.

21. Legs Hédou et Garetta : voir la chronique de Georges Dubosc dans le Journal de Rouen en date du 7 novembre 1926.

22. Pour exemple, la collection de Christophe-Paul de Robien, saisie en 1791 (aujourd'hui répartie entre musée des Beaux-Arts de Rennes, le musée de Bretagne et l'université de Rennes), contient de nombreuses académies.

23. DÉZALLIER d'ARGENVILLE Antoine-Nicolas, Vies des fameux sculpteurs depuis la renaissance des arts, avec la description de leurs ouvrages, Paris, Debure l'aîné, 1787, vol.2, p. 399 [disponible en ligne], https://gallica.bnf.fr/ark:/12148/bpt6k108162h/f439.item [lien valide en janvier 2021].

24. GAEHTGENS Thomas W. \& LUGAND Jacques, Joseph-Marie Vien, peintre du roi. 1716-1809, Paris, Arthena, 1988, p. 113.

25. S. N., Encyclopédie méthodique, tome 1, article «Instruction ", Paris / Liège, Panckoucke / Plamtoux, 1788, p. 456-457.

26. MERCIER Louis-Sébastien, De la littérature et des littérateurs. Suivi d'un Nouvel examen de la tragédie françoise, Yverdon, s. n., 1778, p. 157.

27. RESTOUT Jean-Bernard, «Adresse, mémoires et observations présentés à l'Assemblée nationale le 17 avril 1791 par la Commune des arts qui ont le dessin pour base ", manuscrit rédigé à Paris (Paris, BnF, collection Deloynes, vol. 53, n 1497, n. 9, fo 660-661), 1791, p. 22 [disponible en ligne], https://gallica.bnf.fr/ark:/12148/btv1b10537776m/f3.item [lien valide en janvier 2021]. 28. QUATREMÈRE de QUINCY Antoine, Considérations sur les arts du dessin en France, suivies d'un plan d'Académie, ou d'École publique, et d'un système d'encouragements, Paris, Desenne, 1791, p. 127 [disponible en ligne], https://gallica.bnf.fr/ark:/12148/bpt6k1040218n/f15.item [lien valide en janvier 2021].

29. CONVENTION NATIONALE, Procès-verbaux du Comité d'instruction publique de la Convention nationale. Publiés et annotés par James Guillaume III, 15 octobre 1792-2 juillet 1793, vol.1, Paris, L'Harmattan, 1997-1998, p. 192.

30. RESTOUT Jean-Bernard, Adresse, mémoires et observations...

31. Journal de la Société populaire et républicaine des arts, séance du 13 germinal an II [ 2 avril 1794], p. 323.

32. LENOIR Alexandre, Histoire des arts en France, prouvée par les monumens, suivie d'une description chronologique des statues en marbre et en bronze, bas-reliefs et tombeaux des hommes et des femmes célèbres, réunis au Musée impérial des monumens français, Paris, chez l'auteur, 1810, p. XXVIII.

33. STRUCKMEYER Nina, "Dans l'atelier des élèves de Jacques-Louis David », in NERLICH France \& BONNET Alain (dir.), Apprendre à peindre. Les ateliers privés à Paris, 1780-1863, actes de colloque (Tours, 16-17 juin 2011), Tours, Presses universitaires François-Rabelais, coll. «Perspectives historiques », 2013, p. 117.

34. Voir à ce sujet RIONNET Florence, L'Atelier de moulage du musée du Louvre. 1794-1928, Paris, Réunion des musées nationaux, coll. « Notes et documents des musées de France », 1996. 
35. JOLY Morwena, «La formation des artistes pendant la Révolution. Disséquer "dans la salle d 'anatomie, près de celle des Antiques, cour du Louvre" ", Revue de l'art, 2013, n 180, vol.2, p. 53-59.

36. Voir JOLY Morwena, «La formation des artistes pendant la Révolution... » : de nombreux pensionnaires de l'Académie de Rome sont concernés, tel Réattu, qui songe à réaliser un traité d'anatomie, Girodet, et même Fabre ou Suvée.

37. MORVAN BECKER Frédéric, "L'École gratuite de dessin de Rouen, ou la formation des techniciens au XVIII ${ }^{\mathrm{e}}$ siècle ", thèse d'histoire soutenue à université de Paris-VIII (Saint-Denis), 2010 , p. 372.

38. INGRES Jean-Auguste-Dominique, Écrits et propos sur l'art, Paris, Hermann, coll. "Savoirs ", série « Arts », 2006, p. 175.

39. ESCHOLIER Raymond, Matisse, ce vivant, Paris, Fayard, 1956, p. 21.

40. Alexandre Desachy (ou de Sachy) est mouleur de l'École des beaux-arts de 1848 à 1886 . Voir à ce sujet PINATEL Christiane, «La "Restauration" en plâtre de deux colonnes du temple de Castor et Pollux dans la Petite Écurie royale de Versailles : histoire et archéologie », Revue archéologique, 2003, n 35, vol.1, p. 67-114 [disponible en ligne], https://www.cairn.info/revuearcheologique-2003-1-page-67.htm [lien valide en janvier 2021].

41. MONTENS Valérie, "La création d'une collection nationale de moulages en Belgique. Du musée des Plâtres à la section d'Art monumental des Musées royaux des arts décoratifs et industriels à Bruxelles ", In Situ. Revue des patrimoines [en ligne], $\mathrm{n}^{\circ} 28$, «Le moulage. Pratiques historiques et regards contemporains ", 2016, https://journals.openedition.org/insitu/12564 [lien valide en janvier 2021].

42. BONNET Alain, L'Enseignement des arts au XIX siècle. La réforme de l'École des beaux-arts de 1863 et la fin du modèle académique, Rennes, Presses universitaires de Rennes, 2006, p. 56.

43. ZOLA Émile, L'Éuvre, Paris, Fasquelle, 1967 [1886], p. 17 et 330.

44. BALZAC Honoré de, Le Chef-d'œuvre inconnu, Paris, Éditions de Minuit, 1985 [1831], p. 139.

45. BLAVET Émile, «Les modèles femmes ", La Vie parisienne, 1884, p. 119-124.

46. BONNET Alain, L'Enseignement des arts au XIX siècle, p. 307.

47. JOLY Morwena, La Leçon d'anatomie, p. 184-187.

48. COMAR Philippe (dir.), Figures du corps. Une leçon d'anatomie à l'École des beaux-arts, catalogue d'exposition (Paris, École nationale supérieure des beaux-arts, 21 octobre 2008-4 janvier 2009), Paris, Beaux-Arts, 2008, p. 42-47.

49. «Lettre aux jeunes artistes" (en date du 25 décembre 1861), in COURBET Gustave, Correspondance de Courbet, Paris, Flammarion, 1996, p. 184.

50. PLANCHE Gustave, Portraits d'artistes. Peintres et sculpteurs, vol. 1, Paris, Michel Lévy frères, 1853, p. 162.

51. Gustave Courbet, L'Homme à la ceinture de cuir [portrait de l'artiste entre 1845 et 1846] (Paris, musée d'Orsay).

52. Gustave Moreau est un enseignant très apprécié, dont l'atelier, aujourd'hui transformé en musée (Paris, $9^{\mathrm{e}}$ ), conserve à peu près tous les modèles écorchés en ronde-bosse alors disponibles sur le marché.

53. COQUIOT Gustave, Paul Cézanne, Paris, Librairie Paul Ollendorff, [1919], p. 22.

54. JEAN Raymond, Cézanne, la vie, l'espace, Paris, Éditions du Seuil, coll. « Fiction \& Cie », 1986, p. 96.

55. Entretiens avec Pierre Cabanne, p. 33, cité par MARCADÉ Bernard, Marcel Duchamp. La vie à crédit. Biographie, Paris, Flammarion, coll. « Grandes biographies », 2007, p. 23.

56. «À propos de moi-même ", Marcel Duchamp, Duchamp du signe. Écrits, Paris, Flammarion, 1976, p. 222, cité par MARCADÉ Bernard, Marcel Duchamp, p. 53.

57. Lettre de Marcel Duchamp à Maria Martins du 19 mars [1949], cité par MARCADÉ Bernard, Marcel Duchamp, p. 292. 
58. BROWNE HARE Denise, "Au sujet de l'atelier de Duchamp ", Étant donné n³, p. 48, cité par MARCADÉ Bernard, Marcel Duchamp, p. 496.

59. LANDOWSKI Paul, Peut-on enseigner les beaux-arts ?, Paris, Baudinière, 1943, p. 187.

60. LANDOWSKI Paul, Peut-on enseigner les beaux-arts?, p. 190.

61. Paris, musée d'Orsay, conservé au musée Matisse de Nice.

62. MATISSE Henri, Écrits et propos sur l'art, Paris, Hermann, coll. « Savoir », 1972, p. 163.

63. BÉGOC Janig, « Entre émulation et instrumentalisation. La place du Body Art américain dans la constitution de l'art corporel en France ", in BÉGOC Janig, BOULOUCH Nathalie \& ZABUNYAN Elvan (dir.), La Performance. Entre archives et pratiques contemporaines, Rennes, Presses universitaires de Rennes, coll. « Art \& société », 2010, p. 66.

\section{RÉSUMÉS}

Le patrimoine pédagogique conservé dans les écoles d'art issues des anciennes écoles académiques aurait tendance à laisser penser que l'essentiel de la transmission se joue dans la copie d'après les modèles, antiques ou modernes. C'est oublier qu'au fondement de la pédagogie du dessin telle qu'elle est conçue en France se trouve l'étude d'après le modèle vivant. Une école de l'observation de la nature qui n'a cessé de lutter contre l'hégémonie du modèle antique favorisée par les moulages produits en importante quantité au xIX ${ }^{\mathrm{e}}$ siècle. Il est primordial de connaître l'histoire de ce patrimoine absent avant de concevoir une éventuelle muséographie du patrimoine des écoles d'art.

The pedagogical heritage that is kept in the art schools that have existed for a long time as academies would tend to let us think that most of the drawing practice transmission is done by copying from models, either antique or modern. To believe this is to forget that studying from models is at the core of drawing pedagogy as we conceive it in France. This study by observation of nature has been in a constant struggle against the hegemony of the antique model favoured by the great quantity of plaster casts that was produced in the $19^{\text {th }}$ century. It is of a primordial importance to know the history of that missing heritage before we conceive any potential museography of the heritage of art schools.

\section{INDEX}

Keywords : live model, heritage, art school, art anatomy, antique, pedagogy

Mots-clés : modèle vivant, patrimoine, école d'art, anatomie artistique, antique, pédagogie

\section{AUTEUR}

\section{MORWENA JOLY-PARVEX}

Conservatrice du patrimoine, Centre des Monuments nationaux morwena.joly-parvex@monuments-nationaux.fr 